\title{
Association of CRTC2 gene polymorphisms with growth and meat quality traits of Qinchuan cattle
}

\author{
H.C. Xu ${ }^{1 *}$, L.S. Gui ${ }^{1 *}$, N. Song ${ }^{1}$, Y.Y. Zhang ${ }^{1}$, H.C.Wang ${ }^{1}$ and L.S. Zan ${ }^{1,2}$ \\ ${ }^{1}$ College of Animal Science and Technology, Northwest A\&F University, Yangling, \\ Shaanxi, China \\ ${ }^{2}$ National Beef Cattle Improvement Center of Northwest A\&F University, Yangling, \\ Shaanxi, China
}

*These authors contributed equally to this study.

Corresponding author: L.S. Zan

E-mail: zanlinsen@163.com

Genet. Mol. Res. 14 (4): 12912-12920 (2015)

Received May 11, 2015

Accepted August 26, 2015

Published October 21, 2015

DOI http://dx.doi.org/10.4238/2015.October.21.12

ABSTRACT. Growth and meat quality traits play important roles in the evaluation of cattle productivity and are influenced by genetic and environmental factors. CRTC2 is a recently discovered gene related to obesity that may influence fat deposition. The aim of the current study was to detect polymorphisms of bovine CRTC2 and explore their relationships to growth and meat quality in Qinchuan cattle. Three single nucleotide polymorphisms (SNPs); g.3001 C>T; g.3034 G>A; and g.3467 $\mathrm{T}>\mathrm{C}$, were identified from sequencing results of 422 Qinchuan cattle. The genotypic distributions of both g.3034 G>A and g.3467 T>C mutations were in agreement with Hardy-Weinberg equilibrium, $(P<0.05)$, while the T3001C mutation was not $(P>0.05)$, based on $X^{2}$ test analysis. The SNPs g.3001 C>T and g.3034 G>A are missense mutations (Ser/Phe and Ser/ 
Thr respectively). Additionally, SNPs g.3034 G>A and g.3467 T>C showed a medium polymorphism level $(0.25<\mathrm{PIC}<0.50)$, whereas g.3001 $\mathrm{C}>\mathrm{T}$ showed a low polymorphism level $(\mathrm{PIC}<0.25)$. These three SNPs were significantly associated with several growth and meat quality traits in the Qinchuan cattle population $(P<0.05$ or $P<0.01)$. Collectively, these results demonstrate that CRTC2 is involved in the regulation of cattle growth and meat quality, and suggest that CRTC2 is a potential candidate gene for marker-assisted selection in future breeding development programs for Qinchuan cattle.

Key words: CRTC2; Growth traits; Meat quality traits; Qinchuan cattle; Single nucleotide polymorphism

\section{INTRODUCTION}

Growth and meat quality traits are economically important factors of beef cattle and are controlled by multiple genes (Andrade et al., 2008; Liu et al., 2012). Association studies cannot determine whether gene markers are responsible for variations in a trait, or whether such variation is influenced by closely linked loci. There is, however, a growing body of evidence to suggest that genes directly affect these traits (McGee and Hargreaves, 2010). The need for ongoing improvement in quantitative traits of beef cattle has spurred the development of molecular biotechnology methods, for example, marker-assisted selection, which has emerged as a promising strategy for genetic improvement of growth and carcass traits of beef cattle (Meuwissen and Goddard, 1996). Thus, the relationships between candidate genes and these traits are attracting increasingly more attention.

Target of rapamycin (TOR) is an atypical serine/threonine protein kinase that belongs to the family of phosphatidyl inositol-3 kinase related kinases (PI3K-like kinases or PIKKs). TOR proteins are best known for roles in the nutrient-dependent signaling pathways underlying cell growth, proliferation, and survival (Weisman et al., 2014). TOR genes, which have been isolated in all eukaryotes investigated, form two functionally distinct complexes, TORC1 (CRTC1) and TORC2 (CRTC2); both have been the focus of metabolic and cancer studies for many years (Wullschleger et al., 2006; Loewith and Hall, 2011; Natarajan et al., 2013).

Regulation of transcription of gluconeogenic genes, such as PEPCK and G6Pase, is controlled by AMP-responsive element-binding protein (CREB)-regulated transcription coactivator 2 (CRTC2), which acts by specifying targets for the transcription factor CREB in response to glucagon (Koo et al., 2005). As an important regulator of gluconeogenesis, CRTC2 has a tremendous impact in models of elevated hepatic glucose production. Hepatic glucose production, a focal point of regulation, is repressed during feeding conditions and re-activated upon fasting, in order to meet the fuel requirements for various tissues including brain. CRTC2 can control both glucagon clearance and hepatic amino acid catabolism to regulate glucose metabolism. Studies have shown that CRTC2 knockdown reduced endogenous glucose production and fasting glucose concentrations. Additionally, CRTC2 appears to be a regulator of skeletal muscle oxidative metabolism, consistent with its role as a CREB co-activator (Wang et al., 2009; McGee and Hargreaves, 2010; Erion et al., 2013).

Based on the deduced biological function of CRTC2 in mice and humans, we hypothesized 
that CRTC2 might be associated with cattle growth and meat quality traits. However, polymorphisms in CRTC2 have not yet been reported for cattle. The aim of the current study, therefore, was to investigate single nucleotide polymorphisms (SNPs) in CRTC2 in Qinchuan cattle, to determine possible associations between sequence variation and growth and meat quality traits that may provide genetic markers for Qinchuan cattle breeding.

\section{MATERIAL AND METHODS}

\section{Animal source, DNA samples, and phenotypic data}

Qinchuan beef cattle aged 24-30 months ( $N=422)$ were selected from the Experiment Farm of National Beef Cattle Improvement Center (Yangling, Shaanxi Province, China). In order to detect mutations in CRTC2 in these animals, blood was collected from the jugular vein and stored at $-80^{\circ} \mathrm{C}$, according to the standard phenol chloroform protocol, for DNA extraction (Sambrock and Russell, 2001). Extracted blood DNA content was estimated spectrophotometrically and diluted to $50 \mathrm{ng} / \mathrm{L}$. All DNA samples were stored at $-20^{\circ} \mathrm{C}$ for subsequent analysis.

Eight growth traits of interest were measured and were as follows: body length (BL); wither $(\mathrm{WH})$ and hip $(\mathrm{HH})$ height; rump length $(\mathrm{RL})$; hip width $(\mathrm{HW})$; chest depth $(\mathrm{CD})$; heart girth $(\mathrm{HG})$; and pin bone width (PBW). These traits were measured for statistical analysis as previously described (Gilbert et al., 1993; Brethour, 1994; Hamlin et al., 1995). Two ultrasound traits, ultrasound back fat thickness (UBT) and loin muscle area (ULMA), were evaluated and quantified by ultrasound of live animals.

The above traits were measured according to the criterion: GB/T17238-1998 Cutting Standard of Fresh and Chilled Beef in China (China Standard Publishing House). All experimental procedures were performed under the authorization of the Chinese Ministry of Agriculture.

\section{PCR amplification and sequencing}

Sequence of bovine CRTC2 (GenBank accession No. NM_000160.1) and Primer 5.0 software(PREMIER Biosoft International, Canada) were used to design primer A (sense: 5'-CTTAGTTGCTCCCAGACCAG-3', anti-sense: 5'-ATCCTCATTCCGACAGTACC-3'), to amplify a $1084 \mathrm{bp}$ fragment of CRTC2 in exon and intron 2. Amplification PCR was performed on $20 \mu \mathrm{L}$ mixtures comprising $50 \mathrm{ng}$ sample DNA, $10 \mathrm{pM}$ each primer, $0.20 \mathrm{mM} \mathrm{dNTP,} 2.5 \mathrm{mM} \mathrm{MgCl}_{2}$, and $0.5 \mathrm{U}$ Taq DNA polymerase (TaKaRa; Dalian, China). Amplification reaction conditions were $95^{\circ} \mathrm{C}$ for $5 \mathrm{~min}$; 35 cycles of $94^{\circ} \mathrm{C}$ for $30 \mathrm{~s}, 67.2^{\circ} \mathrm{C}$ for $30 \mathrm{~s}$ (annealing), and $72^{\circ} \mathrm{C}$ for $30 \mathrm{~s}$; and a final extension at $72^{\circ} \mathrm{C}$ for $10 \mathrm{~min}$. Products were digested for detection by electrophoresis on a $1.0 \%$ agarose (w/v) gel, stained with ethidium bromide. Products were then gel-purified using Axygen kits (BMI Fermentas; Glen Burnie, MD, USA), and sequenced in both directions in an ABI PRISM 377 DNA sequencer (Perkin-Elmer). Sequence maps were analyzed using SeqMan 13.0 software (DNASTAR Inc., Madison, Wis.).

\section{Genotyping of CRTC2 alleles by sequencing}

Three novel SNPs were identified in bovine CRTC2 by DNA sequencing: two in exon 2 (g.3001 C>T and g.3034 G>A), and one in intron 2 (g.3467 T>C). All products obtained from 
DNA samples of all cattle used in the study were directly sequenced, in order to distinguish the genotypes of the three mutations in CRTC2.

\section{Statistical analysis}

Gene frequencies, allelic frequencies, and deviations from Hardy-Weinberg equilibrium (HWE) were determined by direct counting. Population genetic indices, including heterozygosity $\left(H_{E}\right)$, effective allele numbers $\left(N_{E}\right)$, and polymorphism information content (PIC) were calculated as previously described (Nei and Roychoudhury, 1974). Trait means were calculated by general linear modeling.

SPSS 19.0 software (SPSS, Inc., Chicago, IL, USA) was used to analyze the relationship between different CRTC2 genotypes and growth and meat quality traits $(B L, W H, H H, R L, H W, C D$, HG, PBW, UBT, ULMA) of Qinchuan cattle. The following statistical linear model was used:

$$
Y i j=\mu+G_{i}+A_{i}+E_{i j k}
$$

$E_{i j k}$ was defined as the trait value for each individual; $\mu$ was the overall population mean for the traits; $G_{i}$ was the fixed effect associated with genotype; $A_{i}$ was the fixed effect of age; and $E_{i j k}$ was the standard error.

\section{RESULTS}

\section{Polymorphisms and genetic diversity}

Polymorphism sites in CRTC2 -g.3001 C>T, g.3034 G>A, and g.3467 T>C were identified by sequencing and were located in exon 2 (g.3001 C>T and g.3034 G>A) and intron 2 (g.3467 T>C). Both g. $3001 \mathrm{C}>\mathrm{T}$ and g.3034 G>A were determined to be missense mutations (Ser/Phe and Ser/Thr).

The different genotypes of each SNP are shown in Figures 1-3. Genotypes CC and CT; GG, AG, and AA; and TT, CT, and CC, were identified by sequencing for SNPs g.3001 C>T, g.3034 $\mathrm{G}>\mathrm{A}$, and g.3467 T>C, respectively. For g.3001 C>T, the TT genotype was not detected in the sampled animals.

\section{Diversity analyses of CRTC2 in the Qinchuan cattle population}

Genotype and allele frequencies were analyzed for the three mutations; results are shown in Table 1. Allele $\mathrm{G}$ was predominant for g.3034 $\mathrm{G}>\mathrm{A}$ and g.3467 T>C, whereas allele $\mathrm{C}$ was predominant for g.3001 C>T. Analysis by $\mathrm{X}^{2}$ testing indicated that HWE was observed at the g.3001 $\mathrm{C}>\mathrm{T}$ and g.3467 $\mathrm{T}>\mathrm{C}$ loci $(\mathrm{P}>0.05)$, whereas individual genotypic frequencies were significantly outside HWE for g.3034 G>A $(P<0.05)$. Heterozygosity values ranged from 0.1322 to 0.4809 and $\mathrm{Ne}$ values from 1.1524 to 1.9265. According to the classification of PIC, SNPs g.3034 G>A and g.3467 T>C showed a medium polymorphism level $(0.25<\mathrm{PIC}<0.50)$, whereas g.3001 C>T showed a low polymorphism level (PIC $<0.25)$. 
H.C. Xu et al.

12916

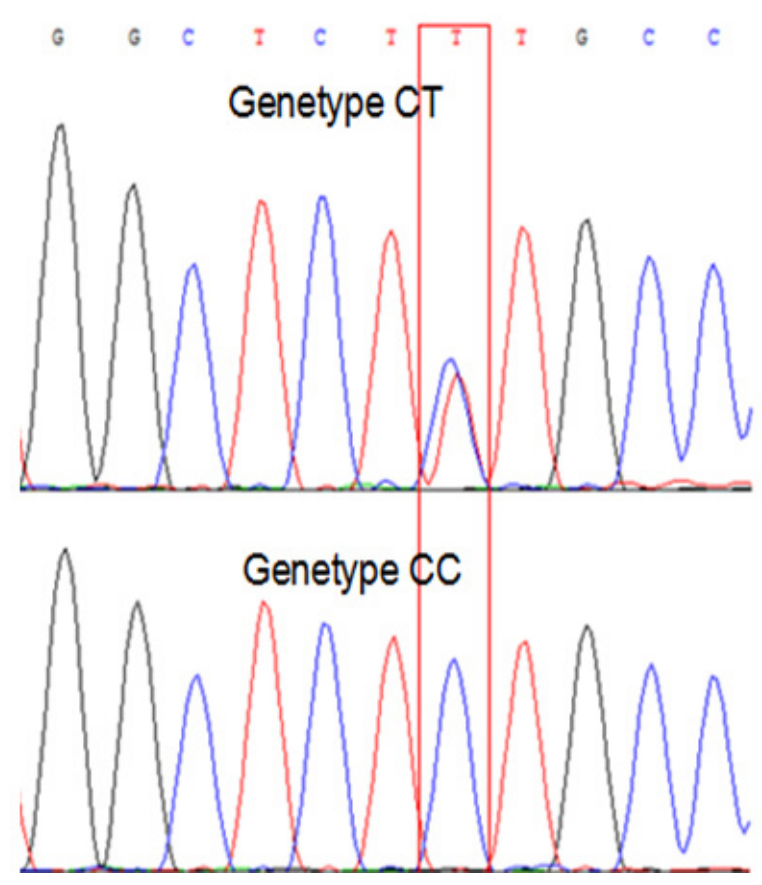

Figure 1. Sequencing maps at g.3001C $>$ T.

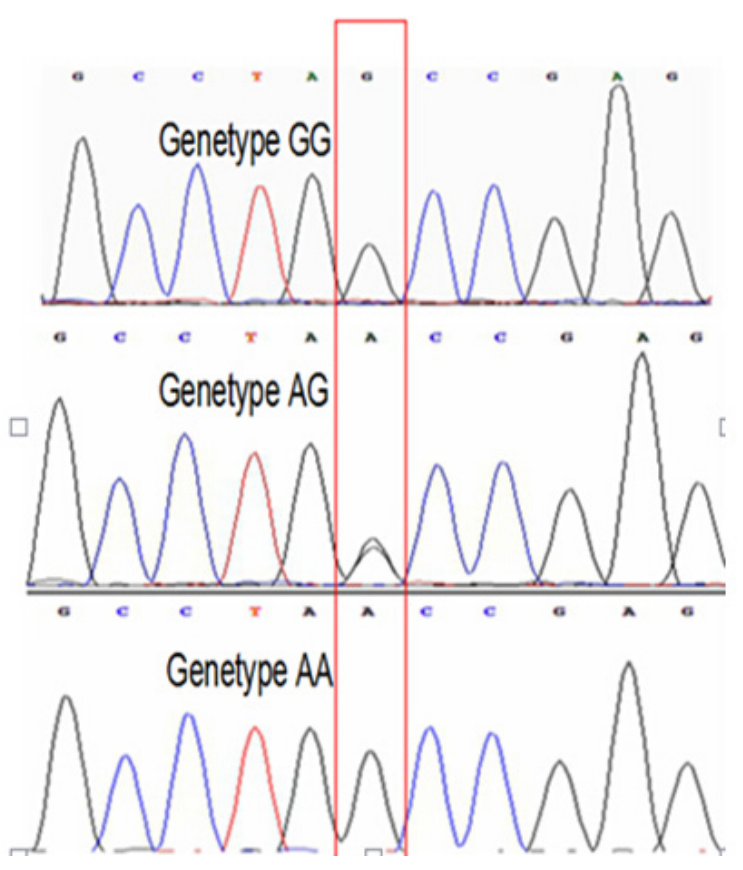

Figure 2 . Sequencing maps at g.3034G>A.

Genetics and Molecular Research 14 (4): 12912-12920 (2015)

CFUNPEC-RP www.funpecrp.com.br 


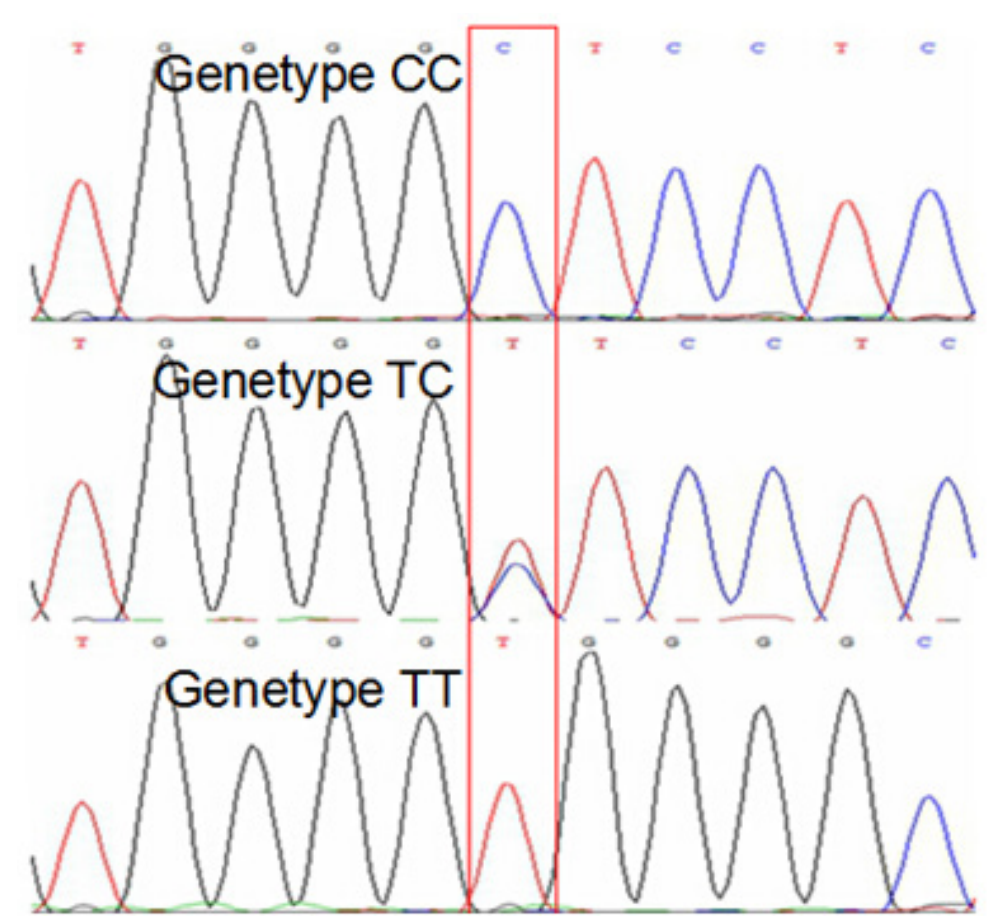

Figure 3. Sequencing maps at g.3467 T>C.

Table 1. Genotypic and allelic frequencies (\%) of CRTC2 gene SNPs in Qinchuan cattle population.

\begin{tabular}{|c|c|c|c|c|c|c|c|c|c|c|}
\hline \multirow{3}{*}{ g. $3001 \mathrm{C}>\mathrm{T}$} & \multirow{2}{*}{$\begin{array}{c}\text { Sample } \\
422\end{array}$} & \multicolumn{3}{|c|}{ Genotypic frequency } & \multicolumn{2}{|c|}{ Allele frequency } & \multirow[t]{2}{*}{$H_{\mathrm{E}}$} & \multirow[t]{2}{*}{$N_{\mathrm{E}}$} & \multirow[t]{2}{*}{$\mathrm{PIC}$} & \multirow[t]{2}{*}{$\mathrm{X}^{2}(\mathrm{HW})$} \\
\hline & & CC & CT & TT & C & $\mathrm{T}$ & & & & \\
\hline & & 0.4615 & 0.4039 & 0 & 0.9288 & 0.0712 & 0.1322 & 1.1524 & 0.1235 & 1.774 \\
\hline \multirow{2}{*}{ g. $3034 \mathrm{G}>\mathrm{A}$} & 422 & GG & AG & AA & G & A & & & & \\
\hline & & 0.5000 & 0.3477 & 0.1523 & 0.6738 & 0.2587 & 0.3272 & 1.7843 & 0.3430 & 13.1942 \\
\hline \multirow[t]{2}{*}{ g. $3467 \mathrm{~T}>\mathrm{C}$} & 422 & $\mathrm{CC}$ & СT & TT & G & A & & & & \\
\hline & & 0.4238 & 0.3477 & 0.1000 & 0.7173 & 0.2827 & 0.4056 & 1.6822 & 0.3233 & 5.101 \\
\hline
\end{tabular}

$\mathrm{HW}=$ Hardy-Weinberg equilibrium; $\mathrm{X}_{0.05}^{2}=5.991, \mathrm{X}_{0.01}^{2}=9.21$

\section{Association of CRTC2 polymorphisms with growth and meat quality traits in Qinchuan cattle}

Effects of CRTC2 SNPs on growth performance and meat quality traits in Qinchuan with genotype $\mathrm{CC}$ had significantly greater $\mathrm{BL}$ and $\mathrm{WH}$, compared to individuals with genotype $C T(P<0.01)$. In addition, statistically significant differences were found in $H H, C D, C C$, and PBW values between these two genotypes $(P<0.05)$. At the g.3034 $G>A$ locus, individuals with genotype GA had greater $\mathrm{BL}, \mathrm{HW}$ and $\mathrm{CD}$ values, compared to those with genotype $\mathrm{GG}$ $(P<0.05)$. At the g.3467 T>C locus, there were highly significant differences in $C C, C D$ and BT values between genotypes TT and GG $(P<0.01)$, and RL, HW, PBW, and ULMA values among cattle with genotype TT were higher than those of cattle with than those with genotype GG $(P<0.05)$. 
H.C. Xu et al.

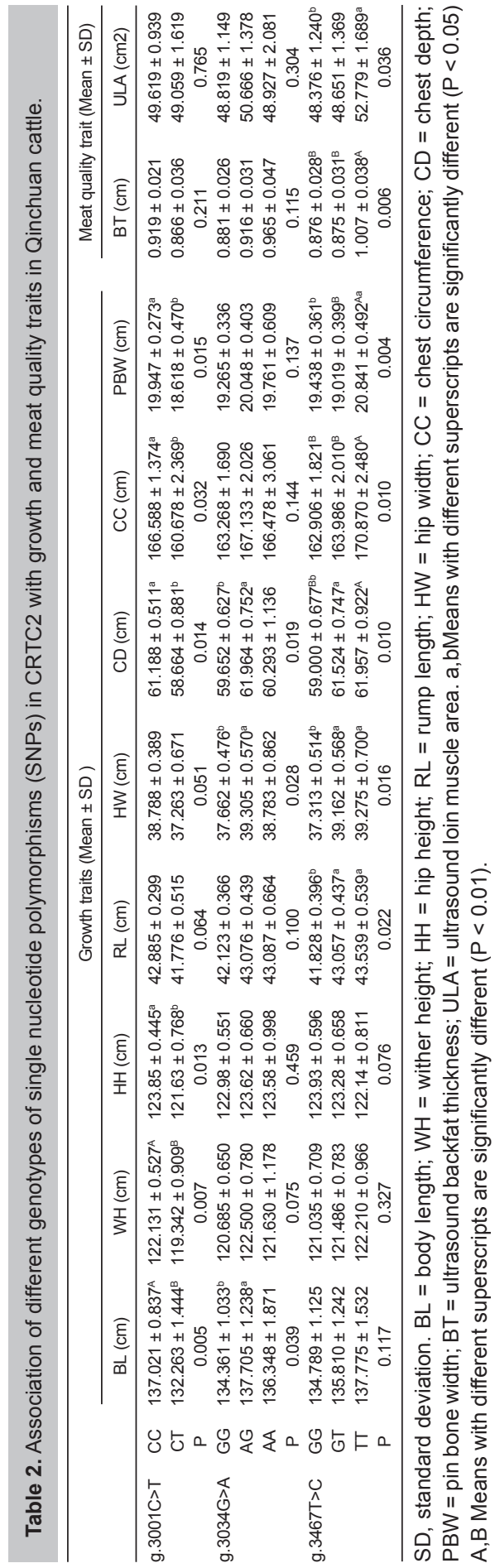




\section{DISCUSSION}

In livestock breeding, growth and meat quality traits are important indices for assessing the economic value of animals that are affected by many factors, including genotype, sex, age, breed, herd, location, and random environmental factors (Liu et al., 2010). Through marker-assisted selection studies, many important genes have been identified that have roles in controlling growth (Xue et al., 2011; Tian et al., 2011) and meat quality in livestock (Jiao et al., 2010; Fan et al., 2011).

Koo et al. (2005) identified CRTC2 as a key regulator for the transcriptional activation of gluconeogenic enzyme, via phosphorylation-dependent cellular localization by AMP-activated protein kinase (AMPK) family of Ser/Thr kinases (Koo et al., 2005). Shaw et al. (2005) reported that adenovirus-mediated inhibition of hepatic CRTC2 reduced PGC-1a expression and normalized blood glucose levels, indicating that CRTC2 is a key target of Liver Kinase B1 (LKB1)/AMP-activated protein kinase (AMPK) signals in the regulation of gluconeogenesis. These studies indicate that CRTC2 may mediate, directly or indirectly, growth and meat production traits in animals.

In this study, the possible relationship between CRTC2 polymorphism and ten growth and meat quality traits was evaluated using 422 Qinchuan cattle. Three SNPs, g.3001 C>T, g.3034 $\mathrm{G}>\mathrm{A}$, and g.3467 T>C, were observed in the 5'-UTR region of CRTC2. Both g.3034 G>A and g.3467 T>C showed three genotypes, whereas g.3001 C>T showed two. Failure to detect the TT genotype for the g.3001 C>T locus may be due to its absence in Qinchuan cattle, or the frequency of this genotype may have been too low to detect in a sample population of the size used in the study. Our data show that the g.3001 C>T SNP influenced BL, WH, HH, CD, CC and PBW values in Qinchuan cattle $(P<0.01$ or $P<0.05)$; the $C C$ genotype appeared to be associated with improved growth and meat quality traits. The g.3034 G>A SNP had significant effects on $\mathrm{BL}, \mathrm{HW}$ and $\mathrm{CD}$ values $(P<0.05)$, and $G A$ seemed to be the beneficial genotype for these traits at this locus. The g.33467 T>C SNP influenced RL, HW, CD, CC, PBW, BT, and ULMA values $(P<0.01$ or $P<0.05)$; GT appeared to be the beneficial genotype.

Glucose homeostasis is tightly regulated in mammals via hormonal or nutritional signals, which govern cellular events in various metabolic tissues (Yoon et al., 2009). Previous reports have concluded that CRTC2 is an important regulator of gluconeogenesis with tremendous impact in models of elevated hepatic glucose production, and regulates transcription of gluconeogenic genes such as PEPCK and G6Pase by specifying targets for the transcription factor CREB in response to glucagon.

Many studies have been conducted on CRTC2 in humans and mice, but few are related to its association with growth and meat quality traits in production animals, and no research in cattle or other livestock species was reported.In this paper, the results presented here provide evidence that CRTC2 affects growth and meat quality traits in Qinchuan cattle populations.Considering the relationship between metabolism and CRTC2, we determined the polymorphism and effect of cattle CRTC2 on growth and meat quality traits.

\section{ACKNOWLEDGMENTS}

Research supported by the National "863" Program of China (grant \#2013 AA102505 and \#2011AA100307-02); National Natural Science Foundation (grant \#31272411); National Beef and Yak Industrial Technology System (grant \#CARS-38); National Science-technology Support Plan Projects (grant \#2012BAD28B04-03); and Technical Innovation Engineering Project of Shaanxi Province (grant \#2014 KTZB02-02-01). 


\section{REFERENCES}

Andrade PC, Grossi DA, Paz CC, Alencar MM, et al. (2008). Association of an insulin-like growth factor 1 gene microsatellite with phenotypic variation and estimated breeding values of growth traits in Canchim cattle. Anim. Genet. 39: 480-485.

Brethour JR (1994). Estimating marbling score in live cattle from ultrasound images using pattern recognition and neural network procedures. J. Anim. Sci. 72: 1425-1432.

Erion DM, Kotas ME, McGlashon J, Yonemitsu S, et al. (2013). cAMP-responsive element-binding protein (CREB)-regulated transcription coactivator 2 (CRTC2) promotes glucagon clearance and hepatic amino acid catabolism to regulate glucose homeostasis. J. Biol. Chem. 288: 16167-16176.

Fan YY, Zan LS, Fu CZ, Tian WQ, et al. (2011). Three novel SNPs in the coding region of PPAR gamma gene and their associations with meat quality traits in cattle. Mol. Biol. Rep. 38: 131-137.

Gilbert RP, Bailey DR and Shannon NH (1993). Linear body measurements of cattle before and after 20 years of selection for postweaning gain when fed two different diets. J. Anim. Sci. 71: 1712-1720.

Hamlin KE, Green RD, Perkins TL, Cundiff LV, et al. (1995). Real-time ultrasonic measurement of fat thickness and longissimus muscle area: I. Description of age and weight effects. J. Anim. Sci. 73: 1713-1724.

Jiao Y, Zan LS, Liu YF, Wang HB, et al. (2010). A novel polymorphism of the MYPN gene and its association with meat quality traits in Bos taurus. Genet. Mol. Res. 9: 1751-1758.

Koo SH, Flechner L, Qi L, Zhang X, et al. (2005). The CREB coactivator TORC2 is a key regulator of fasting glucose metabolism. Nature 437: 1109-11.

Liu Y, Zan L, Zhao S, Xin Y, et al. (2010). Molecular characterization, polymorphism of bovine ZBTB38 gene and association with body measurement traits in native Chinese cattle breeds. Mol. Biol. Rep. 37: 4041-4049.

Liu Y, Liu XL, He H and Gu YL (2012). Four SNPs of insulin-induced gene 1 associated with growth and carcass traits in Qinchuan cattle in China. Genet. Mol. Res. 11: 1209-1216.

Loewith R and Hall MN (2011). Target of rapamycin (TOR) in nutrient signaling and growth control. Genetics 189: 1177-1201.

McGee S and Hargreaves M (2010). AMPK-mediated regulation of transcription in skeletal muscle. Clin. Sci. 118: 507-518.

Meuwissen THE and Goddard ME (1996). The use of marker haplotypes in animal breeding schemes. Genet. Sel. Evol. 28: $161-176$.

Natarajan R, Trivedi-Vyas D and Wairkar YP (2013). Tuberous sclerosis complex regulates Drosophila neuromuscular junction growth via the TORC2/Akt pathway. Hum. Mol. Genet. 22: 2010-2023.

Nei M and Roychoudhury AK (1974). Sampling variance of heterozygosity and genetic distance. Genetics 76: 379-390.

Sambrock J and Russell DW (2001). Molecular cloning: a laboratory manual. 3rd edn. Cold Spring Harbor Laboratory Press, New York.

Tian WQ, Wang HC, Song FB, Zan LS, et al. (2011). Association between a single nucleotide polymorphism in the bovine chemerin gene and carcass traits in Qinchuan cattle. Genet. Mol. Res. 10: 2833-2840.

Weisman R, Cohen A and Gasser SM (2014). TORC2-a new player in genome stability. EMBO Mol. Med. 6: 995-1002.

Wullschleger S, Loewith R and Hall MN (2006). TOR signaling in growth and metabolism. Cell 124: 471-484.

Wang Y, Vera L, Fischer WH and Montminy M. (2009). The CREB coactivator CRTC2 links hepatic ER stress and fasting gluconeogenesis. Nature 460: 534-537.

Xue M, Zan LS, Gao L and Wang HB (2011). A novel polymorphism of the myogenin gene is associated with body measurement traits in native Chinese breeds. Genet. Mol. Res. 10: 2721-2728.

Yoon YS, Ryu D, Lee MW, Hong S, et al. (2009). Adiponectin and thiazolidinedione targets CRTC2 to regulate hepatic gluconeogenesis. Exp. Mol. Med. 41: 577-583. 